\title{
Non-rhizobial endophytes in root nodules
}

\begin{abstract}
Plants of leguminous family are associated specifically with Rhizobium sp. which plays a crucial role in agriculture, forestry and nutrient cycling by maintaining soil fertility through biological nitrogen fixation. The Rhizobia includes genus Rhizobium, Mesorhizobium, Bradyrhizobium, Ensifer and Azorhizobium belongs to $\alpha$ proteobacteria. Despite the high specificity between Rhizobium and legumes, the presence of non-rhizobia in root nodules has been reported. These are belonging to $\alpha, \beta$ and $\delta$-Proteobacteria. This review through a light on diversity and role of these non-rhizoidal species in their host.
\end{abstract}

Keywords: Legume, Non-rhizobial endophytes, plant growth promotion, Rhizobium
Volume 3 Issue I - 2018

\author{
Archana Dhole, Harsha Shelat \\ College of Agriculture, Anand Agricultural University, India
}

Correspondence: Archana Dhole, College of Agriculture, Anand agricultural University, Anand-388I I0, India, Email archanadhole.2009@gmail.com

Received:September 20, 2017 | Published: April 06, 2018

\section{Introduction}

Leguminosae family of plants has more than 19000 species. Currently worldwide revealed that the legumes nodules both wild and cultivated inhabited by Rhizobia. The genus Rhizobium, Mesorhizobium, Bradyrhizobium, Ensifer and Azorhizobium collectively called rhizobial largely attributed to fix atmospheric nitrogen, a major contributor to the global nitrogen cycle. In addition to strains that can elicit nodules root nodules also accommodate various non-rhizobial bacteria having definite influence on the survival, nodulation and grain yield of the crop. ${ }^{1}$ In the absence of positive nodulation tests, they can be regarded as non-rhizobial endophytes (NRE). ${ }^{2}$ In past decade, several non rhizobial symbionts capable of forming nodules and fixing nitrogen in legume roots have been documented and grouped under $\alpha$ and $\beta$-subclass of Proteobacteria, which include Methylobacterium nodulans, Blastobacter denitrificans, Devosia sp., Ochrobactrum lupini, Agrobacterium, Phyllobacterium trifolii, Herbaspirillum lustianum, Ralstonia taiwanensis, Burkholderia tuberum, Burkholderia phymatum, Burkholderia cepacia. Few $\gamma$-proteobacteria (Pantoea, Enterobacter and Pseudomonas). ${ }^{3}$ Actinobacteria (Arthrobacter, Microbacterium and Curtobacterium) and Firmibacteria (Bacillus, Paenibacillus and Staphylococcus) also documented in root nodules. The occurrence of Bacillus species as endophyte has been also reported from different plants such as pigeon pea, wheat, kudzu and soybean nodules. ${ }^{4}$ These NRE bacteria have beneficial effects on the host plants, including plant growth promotion viz. nitrogen fixation and phosphate solubilization and thereby increase the availability of nutrients to synthesize important biomolecules like nucleic acids, amino acids, proteins etc. which is reflected as better plant growth. ${ }^{5-8}$ (Vessey, 2003).Moreover, some are capable of producing IAA which plays a crucial role in plant growth and development by inducing the cell differentiation and regeneration of the vascular tissues and is a regulator of numerous biological processes from cell division, elongation, fruit development, lateral root development, tropistic response and senescence which thereby improve root growth and surface area. In addition, some produce siderophores. ${ }^{9}$ Rajendran et al., (2008) and were also capable of producing stress release enzyme ACC deaminase collectively provides excellent plant growth. Some are involved in biological control of plant pathogens. ${ }^{7}$

\section{Conclusion}

Rhizobia are wide spread soil bacteria able to induce the formation of root nodules and to fix nitrogen in both cultivated and wild leguminous crops. These rhizobia are of economic importance in low input sustainable agriculture, agroforestry and land reclamation. Currently there are five genera of rhizobia in a-Proteobacteria recognized as Azorhizobium, Bradyrhizobium, Rhizobium, Mesorhizobium and Ensifer. ${ }^{3}$ Besides these genera, several $\alpha$ - and $\beta$-Proteobacteria and several unclassified $\delta$-Proteobacteria were recently isolated from nodules of diversified legume plants. These are non nodulating but demonstrate plant growth promotion by different attributes like nitrogen fixation, phosphate solubilization, growth hormone production, Siderophores production and control of pathogens by antagonism.

\section{Acknowledgment}

None.

\section{Conflict of interest}

None.

\section{References}

1. Lin DX, Wang ET, Tang H, et al. Shinella kummerowiae sp. nov., a symbiotic bacterium isolated from root nodules of the herbal legume. Kummerowia stipulacea. Inter J Sys and Evo Micro.2008;58(pt 6):14091413 .

2. Meyer SED, Beuf K D, Vekeman B, et al. A large diversity of non-rhizobial endophytes found in legume root nodules in Flanders (Belgium). Soil Biol \& Biochem. 2015;83:1-11.

3. Balachandar D, Raja P, Kumar K, et al. Non-rhizobial nodulation in legumes. Biotech. and Mol. Bio. Review. 2007;2(2):49-57.

4. Zhao L, Xu Y, Sun R, et al. Identification and characterization of the endophytic plant growth prompter Bacillus cereus strain MQ23 isolated from Sophora alopecuroides root nodules. Brazilian J Micro. 2011;42(2):567-575.

5. Kuklinsky SJ, Araujo WL, Mendes R. Isolation and characterization of soybean-associated bacteria and their potential for plant growth promotion. Env Micro. 2004;6(12):1244-1251. 
6. Ibanez F, Jorge A, Tania T, et al. Endophytic occupation of peanut root nodules by opportunistic Gammaproteobacteria. Sys. and Applied Micro. 2009;32(1): 49-55

7. El-Tarabily KA, Hardy GES, Sivasithamparam K. Performance of three endophytic actinomycetes in relation to plant growth promotion and biological control of Pythium aphanidermatum, a pathogen of cucumber under commercial field production conditions in the United Arab Emirates. European Journal of Plant Pathology. 2010;128:527-539.
8. Tariq M, Hameed S, Yasmeen T, et al. Non-rhizobial bacteria for improved nodulation and grain yield of mung bean [Vigna radiata L. Wilczek]. Afri J Biotech.,2012;11(84):15012-15019.

9. Andrews M, Hodge S, Raven J. Positive plant microbial interactions. Annals of Applied Biology. 2010;157(3):317-320. 